\title{
Sparse connectivity selectively reduces diagnostic facial information: an ICA model of congenital prosopagnosia Rainer Stollhoff*1, Ingo Kennerknecht ${ }^{2}$ and Jürgen Jost ${ }^{1}$
}

\author{
Address: ${ }^{1}$ Max Planck Institute for Mathematics in the Sciences, Leipzig, Germany and ${ }^{2}$ Institut für Humangenetik, Westfälische Wilhelms- \\ Universität, Münster, Germany \\ Email: Rainer Stollhoff* - rainer.stollhoff@mis.mpg.de \\ * Corresponding author
}

from Eighteenth Annual Computational Neuroscience Meeting: CNS*2009

Berlin, Germany. 18-23 July 2009

Published: 13 July 2009

BMC Neuroscience 2009, I0(Supp| I):P36| doi:I0.||86/|47|-2202-|0-SI-P36|

This abstract is available from: http://www.biomedcentral.com/I47I-2202/I0/SI/P36 I

(c) 2009 Stollhoff et al; licensee BioMed Central Ltd.

Congenital prosopagnosia (CP) is defined by a lifelong impairment in face recognition that is present from birth. Subjects with congenital prosopagnosia (CP) show pronounced and selective deficits in facial identification but perform normal in different facial categorization tasks (e.g. recognition of facial expressions). We investigated whether the selectivity of this deficit can be explained by structural ("congenital") differences in the representation and the processing of facial information.

Based on a single-layer neural network model, independent component analysis (ICA) was used to represent the information contained in face images in two different ways. In temporal ICA, a sparseness assumption on the network connectivity leads to distributed face representations in terms of local features. In spatial ICA, sparseness assumptions on output activations produced a factorial face code based on globally dispersed, holistic features.

An experimental comparison of both ICA representations was conducted with a group of $13 \mathrm{CP}$ subjects and 23 agematched controls. Participants had to judge similarity between an average face and a set of test face images manipulated according to one of the two ICA representations. In comparison to control subjects, participants with $\mathrm{CP}$ showed clear deficits in discerning manipulations according to spatial ICA but no significant differences with respect to temporal ICA manipulations.
Based on an ICA model of face representation, our results point to structural differences in the network connectivity as the cause of the face-processing deficit in CP. This hypothesis is supported by a recent imaging study which reveals reduced structural connectivity between ventral regions of the visual cortex in CP subjects [1]. Moreover, a computational study Draper et al. [2] compared the performance of both ICA architectures and observed worse identity discrimination but better emotion discrimination for temporal ICA. Thus, sparse connectivity can selectively reduce facial information diagnostic for identity discrimination.

\section{Acknowledgements}

The authors thank all control and PA subjects for their participation, B. Welling (Uni Münster), T. Elze (MPI MiS), B. Englitz (MPI MiS) I. Bülthoff (MPI Biol. Cyb.) and T. Wolf (MPI Biol. Cyb.) for interesting discussions, technical support and assistance in conducting the experiment.

\section{References}

I. Thomas C, Avidan G, Humphreys K, Jung K, Gao F, Behrmann M: Reduced structural connectivity in ventral visual cortex in congenital prosopagnosia. Nat Neurosci 2009, I 2:29-3I.

2. Draper B, Baek K, Bartlett M, Beveridge J: Recognizing faces with PCA and ICA. Computer Vision and Image Understanding 2003, 91:115-137. 